Submitted for publication in The Astrophysical Journal Supplements

Preprint typeset using LATEX style emulateapj v. 05/12/14

\title{
STAR CLUSTER DISRUPTION IN THE STARBURST GALAXY MESSIER 82
}

\author{
Shuo Li ${ }^{1,2}$, Richard de Grijs ${ }^{2,1}$, Peter Anders ${ }^{3}$, And Chengyuan Li ${ }^{1,2}$ \\ Submitted for publication in The Astrophysical Journal Supplements
}

\begin{abstract}
Using high-resolution, multiple-passband Hubble Space Telescope images spanning the entire optical/near-infrared wavelength range, we obtained a statistically complete sample, $U$-band selected sample of 846 extended star clusters across the disk of the nearby starburst galaxy M82. Based on careful analysis of their spectral energy distributions, we determined their galaxy-wide age and mass distributions. The M82 clusters exhibit three clear peaks in their age distribution, thus defining a relatively young, $\log \left(t \mathrm{yr}^{-1}\right) \leq 7.5$, an intermediate-age, $\log \left(t \mathrm{yr}^{-1}\right) \in[7.5,8.5]$, and an old sample, $\log \left(t \mathrm{yr}^{-1}\right) \geq 8.5$. Comparison of the completeness-corrected mass distributions offers a firm handle on the galaxy's star cluster disruption history. The most massive star clusters in the young and old samples are (almost) all concentrated in the most densely populated central region, while the intermediate-age sample's most massive clusters are more spatially dispersed, which may reflect the distribution of the highest-density gas throughout the galaxy's evolutionary history, combined with the solid-body nature of the galaxy's central region.

Subject headings: globular clusters: general - galaxies: evolution - galaxies: individual (M82) galaxies: star clusters: general - galaxies: star formation
\end{abstract}

\section{INTRODUCTION}

Most current star-formation scenarios are based on the notion that $70 \%-90 \%$ of stars form in embedded clusters (e.g., Lada \& Lada 2003). In their comprehensive review, Portegies Zwart et al. (2010) point out that this is, in fact, supported by the global clustering of spectral O-type stars (Parker \& Goodwin 2007), of which $~ 70 \%$ reside in young clusters or associations (Gies 1987) and $\sim 50 \%$ of the remaining field population are directly identified as runaways (de Wit et al. 2005). Moreover, even some of the merely $\sim 4 \%$ of O-type stars that may not have formed within a clustered environment might indeed also be runaway stars (Gvaramadze \& Bomans 2008; Schilbach \& Röser 2008; Pflamm-Altenburg \& Kroupa 2010). A comparison of the observed formation rate of stars in embedded clusters $\left(\sim 3 \times 10^{3} M_{\odot} \mathrm{Myr}^{-1} \mathrm{kpc}^{-2}\right.$; Lada \& Lada 2003) with stars in the field $\left(\sim 3-7 \times 10^{3} M_{\odot}\right.$ $\mathrm{Myr}^{-1} \mathrm{kpc}^{-2}$; Miller \& Scalo 1979) also strongly supports the notion that clusters embody the fundamental mode of star formation 4

\subsection{Young Massive Star Clusters}

Young massive star clusters (YMCs) are compact regions of very active star formation. Based on analyses of their derived masses, radii, and ages, it is thought

\footnotetext{
1 Department of Astronomy, Peking University, Yi He Yuan Lu 5, Hai Dian District, Beijing 100871, China

${ }^{2}$ Kavli Institute for Astronomy \& Astrophysics, Peking University, Yi He Yuan Lu 5, Hai Dian District, Beijing 100871, China; grijs@pku.edu.cn

${ }^{3}$ Key Laboratory for Optical Astronomy, National Astronomical Observatories, Chinese Academy of Sciences, 20A Datun Road, Chaoyang District, Beijing 100012, China

4 Some level of disagreement persists as to whether stars physically form in star clusters or in more diffuse structures ('fractal distributions,' following the turbulent structure of the interstellar medium; e.g., Gieles et al. 2008; Bastian et al. 2009, 2011; Bressert et al. 2010; Kruijssen 2012; Longmore et al. 2014) that collapse into cluster-like configurations within a few Myr.
}

that YMCs, particularly the most massive and brightest YMCs, could over a Hubble time probably evolve into a population of globular clusters (GCs; e.g., de Grijs \& Parmentier 2007; Portegies Zwart et al. 2010, and references therein).

But can this evolution happen? Meurer et al. (1995) and de Grijs \& Parmentier (2007, and references therein) suggested that any such evolution depends sensitively on the stellar mass distribution, especially on the content of low-mass stars in YMCs. If the stellar initial mass function (IMF) in the clusters is biased toward high-mass stars (a 'top-heavy' IMF), it is unlikely that the clusters can survive the disruptive nature of mass loss due to stellar evolution and dynamical processes (Chernoff \& Shapiro 1987; Chernoff \& Weinberg 1990; Goodwin 1997; Takahashi \& Portegies Zwart 2000; Smith \& Gallagher 2001; Mengel et al. 2002; Kouwenhoven et al. 2014, based on numerical simulations).

YMCs are important objects to study because of the insights they may provide into the formation and destruction of (proto-)GCs (on which we focus in this paper), the star-formation processes in extreme environments, and the triggering and feeding of supergalactic winds (Whitmore et al. 1999; Larsen 2002; Scheepmaker et al. 2007; Hwang \& Lee 2008, 2010; Cantiello et al. 2009; Chandar et al. 2010; Pellerin et al. 2010; Whitmore et al. 2010; Larsen et al. 2011). In addition, their ages and masses can be determined individually through either spectroscopic or multi-passband imaging observations. Hence, they can be used as powerful tracers of the starburst history across a given galaxy. Fortunately, the high spatial resolution of Hubble Space Telescope (HST) observations makes the detection of compact star clusters in starburst regions of several, relatively nearby galaxies feasible.

\subsection{Messier 82}

Recent observations of M82 with the HST/Advanced Camera for Surveys (ACS), covering the entire optical 
extent of the galaxy, offer an excellent opportunity to investigate the role of disruption processes on the cluster mass function (CMF). The shape of the CMF as a function of cluster age is a key diagnostic tool in our quest to understand the evolution of a cluster population in a given galaxy. As one of the nearest galaxies containing a large population of YMCs, the luminosity and size-distribution functions can be studied better in this galaxy than in almost any other galaxy of similar type.

M82 exhibits a biconical, extended filamentary structure (Ohyama et al. 2002). It is the prototype nearby starburst galaxy (e.g., Barker et al. 2008, and references therein) and contains a complex system of star clusters. The energy from these clusters drives the famous $\mathrm{H} \alpha$ and X-ray-bright, kiloparsec-scale superwind (Shopbell \& Bland-Hawthorn 1998; Stevens et al. 2003; Strickland \& Heckman 2007). The M82 system has a very complex structure, which varies on both large and small scales, and its dynamics are also complicated by the inflows and outflows caused by the galaxy's bar and its ongoing starburst.

M82 is a member of the Messier 81 (M81) group. Within the last 200-500 Myr, at least one tidal encounter with M81 occurred. This interaction is thought to be responsible for the triggering of the starburst activity in M82. The strong and varying gravitational effects associated with the most recent M81/M82 flyby have caused the star-formation activity in M82 to increase by an order of magnitude compared with other, 'normal' galaxies. A substantial amount of gas was funneled into the galaxy's core, which resulted in a concentrated starburst, together with a corresponding marked peak in the cluster age distribution. de Grijs et al. (2001) suggested that this starburst may have continued for up to $\sim 50$ Myr at a rate of $\sim 10 M_{\odot} \mathrm{yr}^{-1}$. The core clusters, both YMCs and their less massive counterparts, may have formed during the last of two subsequent starbursts ( $\sim-6$ Myr ago).

In the core of M82, the active starburst region spans a diameter of 500 pc. At optical wavelengths, there are four high-surface-brightness regions or clumps (designated A, C, D, and E by O'Connell \& Mangano 1978), which correspond to known sources at X-ray, infrared (IR), and radio wavelengths. As a result, from our vantage point they are the least obscured starburst complexes. Region A has been studied by many researchers. O'Connell \& Mangano (1978) and O'Connell et al. (1995) suggested that it may be composed of a remarkable complex of YMCs with very high continuum and emission-line surface brightnesses. Shopbell \& Bland-Hawthorn (1998) indicated that the well-known, large-scale bipolar outflow or superwind appears to be centered on regions A and C. Using HST archival data, Melo et al. (2005) found a total of 197 optically visible clusters in the starburst core of M82, with 86 of these residing in region A. Smith et al. (2006) and Westmoquette et al. (2007) used HST/Space Telescope Imaging Spectrograph observations to explore the environment (i.e., the state of the interstellar medium) of the starburst clusters in M82 A and confirm their formation during the most recent starburst event.

Numerous authors have also investigated the properties of the star clusters located outside the central regions. de Grijs et al. (2001, 2003a, 2005) derived pho- tometric ages for the extended, 'fossil' starburst region B located 0.5-1 kpc north-east of the nucleus, showing that a peak of the star-formation activity occurred at $\log \left(t \mathrm{yr}^{-1}\right)=9.0 \pm 0.4$. After a correction due to having previously underestimated the detection limit for wellresolved clusters, Smith et al. (2007) presented new $H S T / A C S U B V I$ photometry for $35 U$-band-selected massive star clusters in the post-starburst region $\mathrm{B}$ of M82. They found that the peak epoch of cluster formation for this sample occurred about $150 \mathrm{Myr}$ ago and star formation continued in M82 B until about 12-20 Myr ago.

The study of Melo et al. (2005) used HST/Wide Field Planetary Camera-2 observations to reveal 197 YMCs in the starburst core (with a mean mass close to $2 \times 10^{5} M_{\odot}$, largely independent of the method used to derive these masses). Mayya et al. (2008) carried out an objective search for star clusters on the HST/ACS images of M82 in the filters F435W $(B), \mathrm{F} 555 \mathrm{~W}(V)$, and F814W $(I)$. Their search led to the discovery of 393 clusters in the disk and 260 clusters in the nuclear region. Lim et al. (2013) used the same data as the present study to analyze the age distribution and the star-formation scenario in M82. Here, we focus on the (tidal) disruption processes in the galaxy and how their influence has shaped the surviving cluster population. This aspect has not yet been covered in any detail for the galaxy as a whole, although a number of studies have considered cluster disruption in spatially confined areas in M82 (e.g., de Grijs et al. 2003c for M82-B). In the present study, a distance of $3.55 \mathrm{Mpc}$, or $(m-M)_{0}=27.75 \pm 0.07 \mathrm{mag}$, is assumed, as determined based on measurements of the $I$-band magnitude of the tip of the red-giant branch (Lim et al. 2013). At the distance of M82, $1^{\prime \prime}$ corresponds to a linear size of $17.2 \mathrm{pc}$.

\section{SOURCE SELECTION AND PHOTOMETRY}

\subsection{Original Data}

The main data set we used was obtained as part of the M82 Hubble Heritage Program (HST proposal GO10776; PI: Mountain). In March 2006, the Hubble Heritage Team observed a large, four-color mosaic image of M82 with the ACS/Wide Field Channel (WFC; pixel size $\sim 0.05^{\prime \prime}$ ) onboard the HST, through the F435W, F555W, $\mathrm{F} 814 \mathrm{~W}$, and $\mathrm{F} 658 \mathrm{~N}$ filters. The latter is a narrow-band filter centered on the $\mathrm{H} \alpha$ emission line. The mosaic image is composed of six separate subimages, with a negligible overlap area. These six 'tiles' were obtained with identical exposure times: each subimage is characterized by four different exposure times of $1600,1360,1360$, and 3320 s. All subimages were combined to reduce the background noise and eliminate cosmic rays (for details, see Mutchler et al. 2007)

A second data set, employing the filters F336W (equivalent to the $U$-band filter; exposure times: 1050, 1215, and $1620 \mathrm{~s}$ ), F110W (roughly equivalent to a combination of the near-IR $Y$ and $J$ bands; exposure times: 598 and $1195 \mathrm{~s}$ ), and F160W (roughly similar to the nearIR $H$ band; exposure times: 598 and 2395 s) has also been included. These latter observations were obtained with the Wide Field Camera 3 (WFC3; proposal GO11360, PI: O'Connell). As opposed to the images from the Hubble Heritage data set, which covers the entire 
galaxy, these image sizes are smaller and only cover part of the galaxy. For all filters, we constructed a combined set of images covering $12288 \times 12288$ pixels $^{2}$, which are fully aligned and have identical pixel sizes of $0.049^{\prime \prime} ; 1$ pixel corresponds to roughly $0.86 \mathrm{pc}$ in linear size. Figure 1 of Lim et al. (2013) shows the overlap areas of the Hubble Heritage data set and the additional data sets composed of images in the F336W, F110W, and F160W filters.

\subsection{Source Selection}

We used a custom-written procedure in IDL to find all relevant sources for further analysis (cf. Barker et al. 2008). As input parameter, the threshold value for detection is related to the number of sources found in applying this procedure. We adopted our images in the HST-equivalent $V$ and $I$ bands as our master images. To decide which minimum (threshold) to use for source detection, for both master images we determined the number of detections as a function of detection threshold. We first estimated the sky (background) noise level pertaining to the image frames in each filter (i.e., the standard deviation in the background count level, determined in regions of the image frames that were largely devoid of even extended galaxy light), $\sigma_{\text {sky }}$, using the IMSTAT routine of the IRAF package 5 We next selected a range of thresholds above the local background (sky + galaxy) level, expressed in units of $\sigma_{\text {sky }}$, i.e., $[2,3,4,5,6] \sigma_{\text {sky }}$ to detect reliable sources. For lower thresholds, the resulting curves are initially steep and then become shallower. We determined the 'knee' in the curve (see Barker et al. 2008), where real sources start to dominate over noise, which occurs for a threshold value of $6 \sigma_{\text {sky }}$ in both passbands. We find that if we vary the local threshold by $\pm 1 \sigma_{\text {sky }}$, the number of detected sources varies by less than $5 \%$. These thresholds correspond to 0.20 and 0.8 counts $\mathrm{s}^{-1}$ in the $V$ and $I$ bands, respectively, resulting in catalogs containing 48,381 objects in the $V$ band and 129,091 objects in the $I$ band.

The next step involved cross-correlation of the source positions in both filters to make sure that we are dealing with real objects in at least these two filters. There are 22,467 sources in common to both images; we adopted maximum offsets between source positions of one pixel in each spatial dimension for a source pair to be considered a match. We subsequently checked the source sizes. To define a minimum size for (extended) cluster candidates, we generated artificial HST point-spread functions (PSFs) using the TINYTIM package (Krist \& Hook 1997) and, based on a PSF comparison, found that sources with observed $\sigma_{\text {Gauss }} \geq 0.84$ pixels are likely to be genuine objects and not spurious features. This selection is safe: the corresponding size cut of 0.84 pixels (intrinsic size convolved with the PSF) is equivalent to Gaussian $\sigma$ of $0.7 \mathrm{pc}$ at the distance of M82. These (marginally to well-resolved) objects must therefore represent genuine clusters instead of individual stars. Inspection of the resulting size distribution shows that half of the M82

\footnotetext{
5 The Image Reduction and Analysis Facility (IRAF) is distributed by the National Optical Astronomy Observatories, which is operated by the Association of Universities for Research in Astronomy, Inc., under cooperative agreement with the U.S. National Science Foundation. We used IRAF version 2.15.1a (February 2011) for the data reduction performed in this study.
}

cluster candidates are 'point' sources, with $\sigma_{\text {Gauss }} \leq 1.1$ pixels. The distribution shows an extended, flat tail out to $\sigma_{\text {Gauss }} \sim 5$ pixels.

\subsection{Photometry}

Using photometry tasks in IDL, we calibrated our source photometry by applying the relevant zero-point offsets based on the values of the image header keywords PHOTFLAM (see Table 1). Using $3.5 \sigma_{\text {Gauss }}$ for the source radii and $[3.5-5] \sigma_{\text {Gauss }}$ for standard sky annulus radii (cf. de Grijs et al. 2013), we obtained aperture photometry for 231 clusters out of 2320 extended cluster candidates identified in total 6 which were present in all of the $U, B, V, I$, and near-IR bands: see Table 2, Compared with the $U B V I$ images, the near-IR data sets are not very sensitive to faint sources, which hence limits the number of objects detected in all filters. In order to have access to a large cluster sample for our subsequent statistical analysis, we also constructed a catalog containing the photometry of the 846 clusters only found in the $U, B, V$, and $I$ bands but not at near-IR wavelengths (see Table 31); the limiting passband in this case is the F336W filter. This latter sample constitutes our master sample of extended star clusters in M82; the cluster properties are included in Table 3 .

\subsection{Completeness Analysis}

In order to obtain a reliable number estimate, we need to assess how many objects may have been missed by our processing technique. We first generated a blank template image without any background noise, with the same size as that of the Hubble Heritage data set. Then, we used the MKOBJ task in IRAF to generate 500 artificial sources with $\sigma_{\text {Gauss }}=1.0$ pixels (representing 'point' sources), which we added to $500 \times 500$ pixel $^{2}$ sections of the blank template at random $(X, Y)$ coordinates, ranging from 0 to 500 pixels in both dimensions. All 500 artificial sources were assigned the same magnitude; we repeated this approach by varying the magnitudes of the artificial sources from 18.0 mag to $28.0 \mathrm{mag}$ for each filter, in steps of 0.5 mag. We added all artificial source frames to both the template and science images. We repeated this procedure to cover the entire template region, thus allowing us to gain a sense of the variability of the completeness levels across the full observational area.

Finally, we 'discovered' the number of artificial sources in both the template and science images, using the same approach for both. (We similarly applied our source discovery routine to the science images to find the real clusters in Section 2.2.) Since the template image contained no background noise, we can recover most input artificial sources, although some artificial sources may have

6 For comparison, Lim et al. (2013) found a total of 1105 star cluster candidates using a combination of automatic source detection and subsequent visual screening. Since that latter step involves a degree of subjectivity, we prefer to base our analysis entirely on automated methods, which we have used successfully in the past (e.g., de Grijs et al. 2003a,b, 2013; Barker et al. 2008; de Grijs \& Anders 2012), although we point out that a visual check done at an early stage of this work resulted in a cluster sample that led to statistically indistinguishable results compared with our automated methods. This is so, because our clusters are sufficiently massive so as not to be unduly affected by stochastic sampling at the fairly close distance to M82. We are striving to reduce the extent of subjectivity in our analysis methods by as much as possible. 
TABLE 1

Detailed observational PRoperties of THE ADOPTED DATA SETS.

\begin{tabular}{|c|c|c|c|c|c|}
\hline Filter & Proposal ID/PI & Camera & Exposure time (s) & Number of images & PHOTFLAM \\
\hline F435W (B) & GO-10776/Mountain & $H S T / \mathrm{ACS}$ WFC & $1360(\times 2), 1600,3320$ & 4 & $3.1412476 \times 10^{-19}$ \\
\hline $\mathrm{F} 555 \mathrm{~W}(V)$ & GO-10776/Mountain & $H S T / A C S$ WFC & $1360(\times 2), 1600,3320$ & 4 & $1.9559270 \times 10^{-19}$ \\
\hline $\mathrm{F} 814 \mathrm{~W}(I)$ & GO-10776/Mountain & $H S T / \mathrm{ACS}$ WFC & $1360(\times 2), 1600,3320$ & 4 & $7.0723600 \times 10^{-20}$ \\
\hline F336W $(U)$ & GO-11360/O'Connell & HST/WFC3 & $1050,1215,1620$ & 3 & $1.3407437 \times 10^{-18}$ \\
\hline $\mathrm{F} 110 \mathrm{~W}(Y J)$ & GO-11360/O'Connell & $H S T / W F C 3$ & 598,1195 & 2 & $1.5232975 \times 10^{-20}$ \\
\hline F160W $(H)$ & GO-11360/O'Connell & HST/WFC3 & 598,2395 & 2 & $1.9106037 \times 10^{-20}$ \\
\hline
\end{tabular}

TABLE 2

Photometry of the 231 M82 Cluster CANDidates Detected in all of the $U B V I(Y J) H$ Passbands.

\begin{tabular}{|c|c|c|c|c|c|c|c|c|c|}
\hline$\left({ }^{\circ}\right)$ & $\begin{array}{l}\text { (J2000) } \\
\text { (hh mm ss.ss) }\end{array}$ & $\left({ }^{\circ}\right)^{\text {Dec. }}$ & $\begin{array}{l}\mathrm{J} 2000) \\
\left({ }^{\circ},{ }^{\prime \prime \prime}\right)\end{array}$ & $\begin{array}{c}m_{\mathrm{F} 336 \mathrm{~W}} \\
(\mathrm{mag})\end{array}$ & $\begin{array}{c}m_{\mathrm{F} 435 \mathrm{~W}} \\
(\mathrm{mag})\end{array}$ & $\begin{array}{c}m_{\mathrm{F} 555 \mathrm{~W}} \\
(\mathrm{mag})\end{array}$ & $\begin{array}{c}m_{\mathrm{F}} 814 \mathrm{~W} \\
(\mathrm{mag})\end{array}$ & $\begin{array}{c}m_{\mathrm{F} 110 \mathrm{~W}} \\
(\mathrm{mag})\end{array}$ & $\begin{array}{c}m_{(\mathrm{F} 160 \mathrm{~W}} \\
(\mathrm{mag})\end{array}$ \\
\hline 148.99374 & 095558.50 & 69.685612 & $\begin{array}{llll}69 & 41 & 08.20\end{array}$ & $22.44 \pm 0.62$ & $20.82 \pm 0.17$ & $21.06 \pm 0.15$ & $21.45 \pm 0.12$ & $22.62 \pm 0.07$ & $22.65 \pm 0.07$ \\
\hline 148.99331 & $09 \quad 55 \quad 58.39$ & 69.685324 & $\begin{array}{llll}69 & 41 & 07.17\end{array}$ & $22.17 \pm 0.56$ & $23.41 \pm 0.48$ & $23.27 \pm 0.37$ & $23.31 \pm 0.23$ & $22.51 \pm 0.16$ & $22.53 \pm 0.15$ \\
\hline 148.99044 & $09 \quad 55 \quad 57.71$ & 69.684583 & $\begin{array}{llll}69 & 41 & 04.50\end{array}$ & $19.69 \pm 0.18$ & $20.51 \pm 0.16$ & $20.81 \pm 0.17$ & $22.07 \pm 0.15$ & $22.63 \pm 0.13$ & $22.66 \pm 0.13$ \\
\hline 148.98999 & $09 \quad 55 \quad 57.60$ & 69.686996 & $\begin{array}{lll}69 & 41 & 13.19\end{array}$ & $24.29 \pm 1.49$ & $24.68 \pm 1.04$ & $24.21 \pm 0.56$ & $23.39 \pm 0.22$ & $26.11 \pm 0.44$ & $26.12 \pm 0.44$ \\
\hline 148.98958 & $09 \quad 55 \quad 57.50$ & 69.684892 & $\begin{array}{llll}69 & 41 & 05.61\end{array}$ & $21.43 \pm 0.39$ & $22.25 \pm 0.42$ & $22.45 \pm 0.32$ & $23.38 \pm 0.31$ & $26.12 \pm 0.59$ & $26.12 \pm 0.59$ \\
\hline . & . . & . & $\cdots$ & $\cdots$ & $\cdots$ & $\cdots$ & $\cdots$ & . . & . . \\
\hline
\end{tabular}

Note. - Table 2 is published in its entirety in the electronic edition of The Astrophysical Journal Supplements. A portion is shown here for guidance regarding its form and content.

TABLE 3

OBSERVED AND DERIVED PROPERTIES OF THE $U B V I$-BASED SAMPLE OF CANDIDATE M82 Clusters.

\begin{tabular}{|c|c|c|c|c|c|c|c|c|c|c|c|}
\hline \multicolumn{2}{|c|}{ R.A. (J2000) } & \multicolumn{2}{|c|}{ Dec. (J2000) } & \multirow{2}{*}{$\begin{array}{c}m_{\mathrm{F} 336 \mathrm{~W}} \\
\text { (mag) }\end{array}$} & \multirow{2}{*}{$\begin{array}{c}m_{\mathrm{F} 435 \mathrm{~W}} \\
(\mathrm{mag})\end{array}$} & \multirow{2}{*}{$\begin{array}{c}m_{\mathrm{F} 555 \mathrm{~W}} \\
\text { (mag) }\end{array}$} & \multirow{2}{*}{$\begin{array}{c}m_{\mathrm{F} 814 \mathrm{~W}} \\
(\mathrm{mag})\end{array}$} & \multirow{2}{*}{$\begin{array}{c}\log (t) \\
{[\mathrm{yr}]}\end{array}$} & \multirow{2}{*}{$\begin{array}{c}\log \left(M_{\mathrm{cl}}\right) \\
{\left[M_{\odot}\right]}\end{array}$} & \multirow{2}{*}{$\begin{array}{c}E(B-V) \\
(\mathrm{mag})\end{array}$} & \multirow{2}{*}{$\begin{array}{l}R_{\mathrm{hl}} \\
(\mathrm{pc})\end{array}$} \\
\hline$\left({ }^{\circ}\right)$ & (hh mm ss.ss) & $\left({ }^{\circ}\right)$ & $\left({ }^{0}{ }^{\prime \prime \prime}\right)$ & & & & & & & & \\
\hline 148.87847 & $09 \quad 55 \quad 30.83$ & 69.665917 & 693957.30 & $24.22 \pm 1.41$ & $24.18 \pm 0.64$ & $23.38 \pm 0.33$ & $23.47 \pm 0.20$ & $8.55_{-0.33}^{+0.47}$ & $5.58_{-0.36}^{+0.45}$ & 0.00 & 2.52 \\
\hline 148.88296 & 095531.91 & 69.670109 & $\begin{array}{lll}69 & 40 & 12.39\end{array}$ & $28.57 \pm 1.88$ & $25.77 \pm 1.36$ & $25.14 \pm 0.75$ & $24.20 \pm 0.27$ & $\begin{array}{r}8.49_{-0.46}^{+0.68} \\
\text { (n) }\end{array}$ & $5.05_{-0.52}^{+0.66}$ & 1.00 & 0.83 \\
\hline 148.89413 & 095534.59 & 69.676876 & $\begin{array}{llll}69 & 40 & 36.75\end{array}$ & $24.25 \pm 1.43$ & $23.68 \pm 0.50$ & $23.77 \pm 0.39$ & $24.29 \pm 0.27$ & $7.76_{-0.52}^{+0.58}$ & $4.70_{-0.56}^{+0.6 \overline{2}}$ & 0.30 & 2.87 \\
\hline 148.89483 & $09 \quad 5534.76$ & 69.671262 & $69 \quad 40 \quad 16.54$ & $29.11 \pm 1.88$ & $26.35 \pm 1.86$ & $25.65 \pm 0.99$ & $24.13 \pm 0.27$ & $8.21_{-0.62}^{+0.65}$ & $5.13_{-0.71}^{+0.73}$ & 1.30 & 0.97 \\
\hline 148.89570 & $0955 \quad 34.97$ & 69.670507 & $\begin{array}{lll}69 & 40 & 13.83\end{array}$ & $24.29 \pm 1.46$ & $23.63 \pm 0.52$ & $24.11 \pm 0.49$ & $24.35 \pm 0.34$ & $7.72_{-0.29}^{+0.34}$ & $3.96_{-0.34}^{+0.35}$ & 0.30 & 3.41 \\
\hline ... & ... & & $\ldots$ & $\cdots$ & . & & & & & . & $\cdots$ \\
\hline
\end{tabular}

Note. - Typical uncertainties in the total (foreground + internal) extinction, $E(B-V)$ are 0.05 to 0.10 mag; typical uncertainties in $R_{\mathrm{hl}}$ are 0.02 to $0.05 \mathrm{pc}$.

Table 3 is published in its entirety in the electronic edition of The Astrophysical Journal Supplements. A portion is shown here for guidance regarding its form and content.

disappeared into the physical noise of the science images, depending on the combinations of their integrated magnitudes and extent, as well as on the real background variations. In addition, saturated sources and blending may cause failures to recover the artificial sources. The template image hence became a perfect comparison sample to check how many sources should be recovered under ideal conditions. We counted the number of recovered artificial sources (from the science image) and the number of recovered artificial sources (from the template) to estimate the completeness, i.e. $f_{\text {comp }}=N_{\text {rec }} / N_{\text {tot }}$, where $N_{\text {rec }}$ is the number of artificial objects we recovered and $N_{\text {tot }}$ is the number of input objects; $f_{\text {comp }}$ was simultaneously corrected for the effects of blending and saturation, as well as background noise. We show the results for the full science observations, as a function of magnitude, in Figure 1. We found that, for the observational data set as a whole, at approximately $21.5 \mathrm{mag}$ in the F336W band, $23 \mathrm{mag}$ in $\mathrm{F} 555 \mathrm{~W}$, and $23.5 \mathrm{mag}$ in the $\mathrm{F} 110 \mathrm{~W}$ band, the completeness fraction drops to below $50 \%$. This indicates that at those magnitudes, the background noise starts to dominate the image quality. In the remainder of this paper, we will only consider objects above their respective $50 \%$ completeness limits and correct the relevant numbers for sampling incompleteness. Note that, strictly speaking, this relates to the cluster subsample representing point sources, which thus implies that the completeness levels derived here are upper limits to the complement of more extended cluster candidates. However, in practice the completeness levels derived here differ only marginally (and firmly within the photometric uncertainties) from those for objects with $\sigma_{\text {Gauss }} \in[2,3]$ pixels; only $\sim 15 \%$ of M82 cluster candidates are more extended.

\section{CLUSTER EVOLUTION}

\subsection{Parameter Determination}

In Section 2 we obtained the observed (broad-band) spectral-energy distributions (SEDs). A small fraction of our sample (231 clusters) were covered in all of the $U, B, V, I$, and near-IR bands, whereas a larger cluster sample of 846 objects was found only in the $U B V I$ bands. We used the GALEV evolutionary synthesis models (Kotulla et al. 2009, and references therein; http://www.galev.org) to determine the best-fitting values for the ages, masses, extinction values, and metallicities for all clusters by comparing the set of model SEDs with our observed SEDs. Our statistical comparison was based on application of the ANALYSED tool, which has been tested extensively both internally (de Grijs et al. 


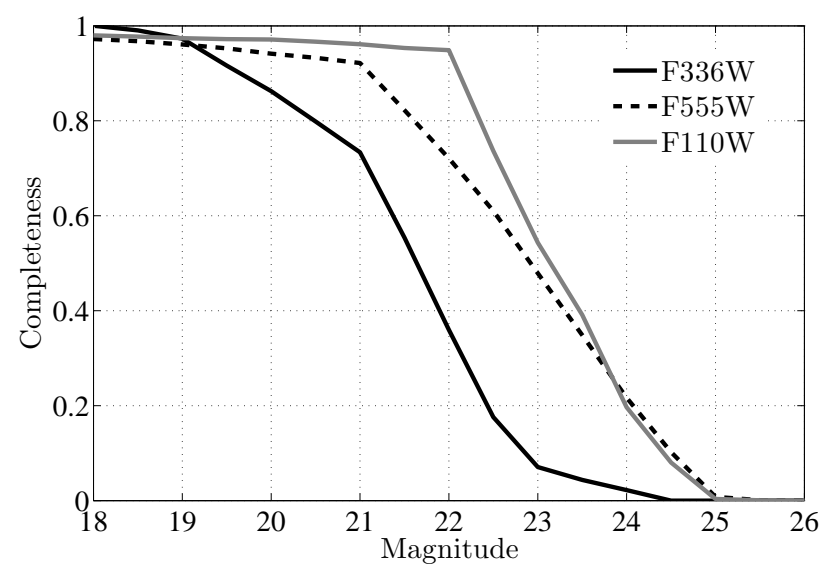

FIG. 1.- Completeness curves for the cluster sample obtained from the HST/WFC3 and ACS images. The analysis was done for all data in the F336W, F555W, and F110W filters.

2003a,b; Anders et al. 2004b) and externally (de Grijs et al. 2005), using both theoretical and observed young to intermediate-age $\left(\leq 3 \times 10^{9}\right.$ yr-old $)$ star cluster SEDs.
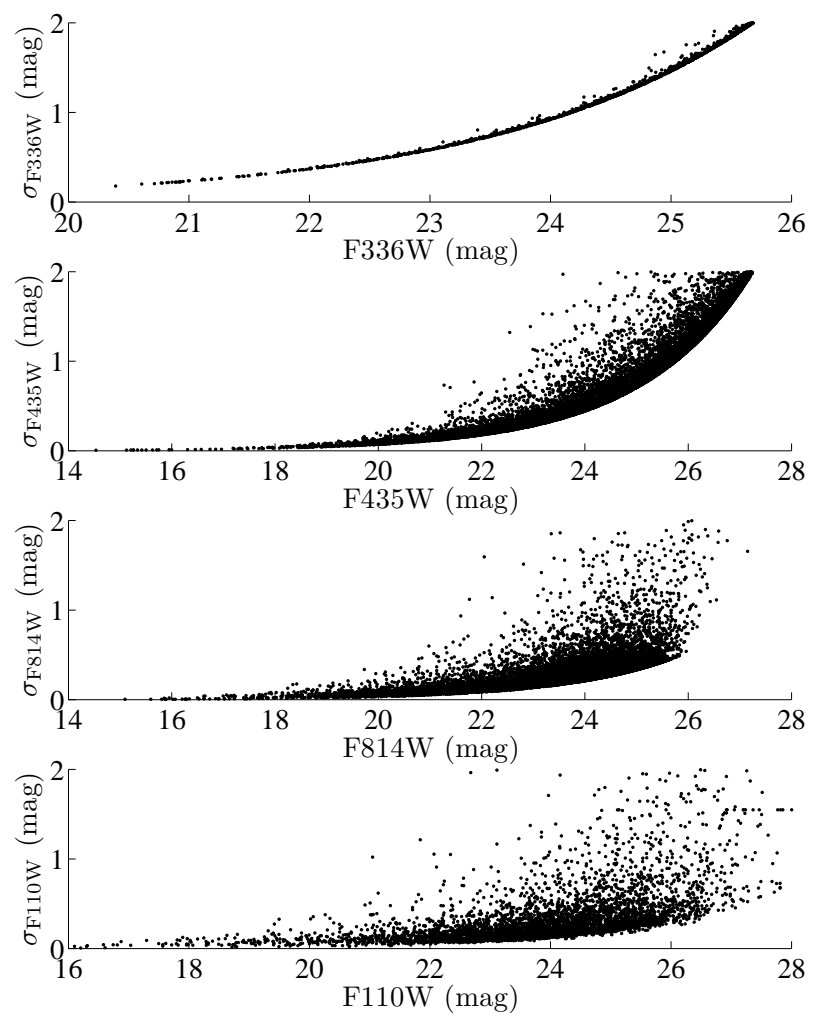

FIG. 2.- Photometric uncertainties as a function of integrated cluster magnitude for the F336W, F435W, F814W, and F110W filters.

The relative ages and masses within a given cluster system can be determined to very high accuracy, provided that a combination of at least four passbands is used as input parameters (Anders et al. 2004b). We first applied the ANALYSED approach to our smaller set of broad-band $U B V I(Y J) H$ cluster SEDs to determine the best-fitting overall metallicity, which yielded $Z=0.02$ (solar metallicity). Based on abundance measurements of HiI regions and young clusters in M82 (Smith et al. 2006; Lançon et al. 2008), solar metallicity for the star clusters seems indeed reasonable. We thus decided to fix the model cluster metallicities to the solar value, leaving both the cluster ages and extinction values as free parameters. The advantage of this approach is that this leaves us with one fewer free parameter to determine, which in turn renders our resulting age, mass, and extinction estimates more robust. The shape of the broad-band SEDs constrains the ages and extinction values, whereas the absolute flux levels result in the corresponding cluster masses. Despite the sometimes significant photometric uncertainties (see Fig. 22), generally caused by the highly variable background, our SED-matching approach successfully converged to a reasonably well-determined set of ages (cf. Table 3).

The F336W band is the filter limiting our completeness analysis; it reaches the $50 \%$ detection limit at a magnitude of 21.5. Although the M82 starburst regions are pervaded by high-extinction filaments, the outermost parts of the starburst core have lower extinction and can be studied with optical telescopes. However, the use of optical wavelengths, and in particular the need for a sufficiently high signal-to-noise ratio in the F336W filter, effectively limits our sampling of the M82 starbursts to their surface regions. Using the GALEV model suite, we draw the $50 \%$ completeness limit (for 'point' sources) defined by the F336W band in the $\log ($ age)$\log$ (mass) diagram of Fig. 3 (top). Formally, 691 of our 846 clusters are located above the F336W 50\% completeness limit. In essence, our cluster sample therefore represents a ' $U$-band selected' sample. With this caveat in mind, two clear "gaps" in the clusters' galaxywide age distribution become apparent, which implies a bursty cluster-formation history across the face of the galaxy. The corresponding histogram (see Fig. 3, bottom) shows that the gap at the youngest age is located at $\log \left(t \mathrm{yr}^{-1}\right) \sim 7.5$, while the second gap is located at approximately $\log \left(t \mathrm{yr}^{-1}\right)=8.5$.

We hence divided our star clusters into three samples: the young sample is composed of clusters with ages younger than $10^{7.5} \mathrm{yr}(t \leq 30 \mathrm{Myr})$, the intermediateage sample's member clusters are aged between $10^{7.5} \mathrm{yr}$ and $10^{8.5}$ yr $(30 \mathrm{Myr} \leq t \leq 300 \mathrm{Myr})$, and the old sample hence consists of the remaining clusters, characterized by ages in excess of $10^{8.5} \mathrm{yr}(t \geq 300 \mathrm{Myr})$. The youngest sample thus encompasses the time of the most recent starburst in the galaxy's nucleus, 4-6 Myr ago (e.g., Barker et al. 2008), while the intermediate-age sample may be associated with the burst of cluster formation induced by the last M81/M82 flyby (e.g., de Grijs et al. 2001; Smith et al. 2007).

Lim et al. (2013) separated their sample of more than 1100 clusters by region, and found that each region (specifically, the galaxy's disk, its halo, the nuclear region, and region M82-B) exhibits a different cluster formation history. Here, we are interested in the global characteristics of the M82 cluster population. Somewhat surprisingly given the good match between their and our 
sets of age derivations (see below), Lim et al.'s (2013) overall cluster age distribution exhibits a clear, dominant peak at $\log \left(t \mathrm{yr}^{-1}\right) \sim 8.7$, which is consistent with the age distribution shown in Fig. 3 (bottom); except for the nuclear region, they do not show evidence of any gaps in the age distribution. In contrast, de Grijs et al. (2003c; their Fig. 4c) found a significant gap in the cluster-formation rate in M82-B near $\log \left(t \mathrm{yr}^{-1}\right) \sim 8.5$, a region not selected for specific analysis by Lim et al. (2013).

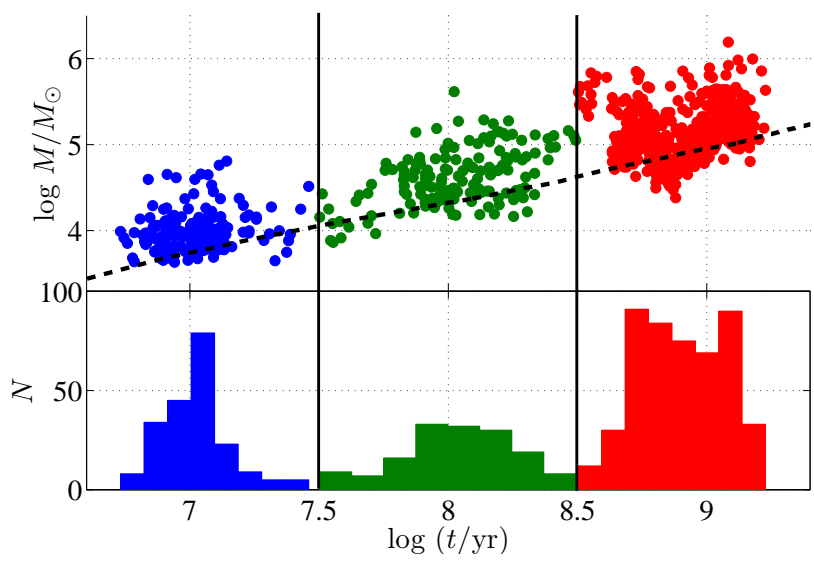

FIG. 3.- Top: Distribution of M82 clusters in the $\log$ (age)$\log$ (mass) plane. The dashed line indicates the F336W band's 50\% completeness level (for 'point' sources). Blue, green, and red data points indicate the youngest, intermediate-age, and oldest cluster samples, respectively. Bottom: Corresponding age distribution.

We compared our photometry and derived cluster ages and masses with the equivalent values of Melo et al. (2005), Smith et al. (2007), and Lim et al. (2013) for 21, eight, and 311 clusters in common, respectively. A quantitative comparison between our values and the relevant samples from the literature is given in Table 4

The majority of the clusters in the intermediate-age and old samples are more massive than a few $\times 10^{4} M_{\odot}$. Based on our recent analysis of the impact of stochastic sampling of stellar mass functions on integrated star cluster properties (Anders et al. 2013; de Grijs et al. 2013; see also Silva-Villa \& Larsen 2011) we conclude that the effects of stochasticity are minimal for M82 clusters that are older than a few $\times 10^{7} \mathrm{yr}$. The context for the clusters in our youngest subsample is different, however: these clusters are all less massive than $10^{5} M_{\odot}$. Nevertheless, the young ages of a subset of these clusters have been confirmed independently on the basis of spectroscopic observations (nuclear region: Smith et al. 2006; Westmoquette et al. 2007; M82-B: Konstantopoulos et al. 2008), as well as by independent analysis of the cluster population in the starburst nucleus (Barker et al. 2008), which also included information about the clusters' $\mathrm{H} \alpha$ luminosities. In addition, the match of our cluster age estimates with the largest comparison sample, that of Lim et al. (2013), is equally good for any age range. This supports our assumption that stochasticity in the clusters' mass functions does not dominate the results presented here (see also the equivalent conclusion in de Grijs et al. 2013), although some level of stochasticity is
TABLE 4

STATISTICAL COMPARISON OF OUR PHOTOMETRY AND DERIVED PARAMETERS WITH RESPECT TO A NUMBER OF KEY COMPARISON SAMPlES. All DIFFERENCES AND STANDARD DEVIATIONS ARE IN THE SENSE "OUR MEASUREMENTS MINUS LITERATURE DATA"; PHOTOMETRIC COMPARISONS ARE IN MAGNITUDES.

\begin{tabular}{|c|c|c|c|}
\hline \multirow[t]{2}{*}{ Parameter } & \multicolumn{3}{|c|}{ Differences with respect to } \\
\hline & $\begin{array}{c}\text { Melo } \\
\text { et al. (2005) }\end{array}$ & $\begin{array}{c}\text { Smith } \\
\text { et al. (2007) }\end{array}$ & $\begin{array}{c}\operatorname{Lim} \\
\text { et al. (2013) }\end{array}$ \\
\hline$N_{\mathrm{cl}}$ & 21 & 8 & 311 \\
\hline \multicolumn{4}{|c|}{ 1. Photometry } \\
\hline$\Delta m_{\mathrm{F} 336 \mathrm{~W}}$ & & & 0.0008 \\
\hline$\sigma_{\mathrm{F} 336 \mathrm{~W}}$ & & & 0.0348 \\
\hline$\Delta m_{\mathrm{F} 435 \mathrm{~W}}$ & -0.010 & 0.025 & 0.0007 \\
\hline$\sigma_{\mathrm{F} 435 \mathrm{~W}}$ & 0.245 & 0.212 & 0.0373 \\
\hline$\Delta m_{\mathrm{F} 555 \mathrm{~W}}$ & -0.010 & 0.088 & 0.0005 \\
\hline$\sigma_{\mathrm{F} 555 \mathrm{~W}}$ & 0.175 & 0.203 & 0.0422 \\
\hline$\Delta m_{\mathrm{F} 814 \mathrm{~W}}$ & -0.095 & 0.100 & 0.0002 \\
\hline$\sigma_{\mathrm{F} 814 \mathrm{~W}}$ & 0.235 & 0.212 & 0.0518 \\
\hline$\Delta m_{\mathrm{F} 110 \mathrm{~W}}$ & & & -0.0001 \\
\hline$\sigma_{\mathrm{F} 110 \mathrm{~W}}$ & & & 0.0501 \\
\hline \multicolumn{4}{|c|}{ 2. Derived parameters } \\
\hline$\Delta \log \left(M_{\mathrm{cl}} / M_{\odot}\right)$ & 0.085 & & \\
\hline$\sigma_{\log } M_{\mathrm{cl}}$ & 0.091 & & \\
\hline$\Delta \log \left(t \mathrm{yr}^{-1}\right)$ & & 0.052 & 0.1668 \\
\hline$\underline{\sigma_{\log t}}$ & & 0.074 & 0.2135 \\
\hline
\end{tabular}

likely to affect both our and Lim et al.'s (2013) results.

Figure 4 shows the mass distribution of the clusters located above the $50 \%(\mathrm{~F} 336 \mathrm{~W})$ completeness limits (for 'point' sources) for all three subsamples. In each panel, the CMF can be reasonably well fitted by a powerlaw CMF of the form $\log N_{\mathrm{cl}} \propto \log \left(M_{\mathrm{cl}}\right)^{-\alpha}$ (dashed lines). The slopes of the dashed lines (obtained by fitting all clusters in the respective samples) are $\alpha=$ $(-2.51 \pm 0.41),(-1.18 \pm 0.43)$, and $(-1.00 \pm 0.18)$ for the young, intermediate-age, and old samples, respectively. The solid lines indicate the theoretically expected slope of $\alpha=-2$ (e.g., Whitmore et al. 1999; Zhang \& Fall 1999).
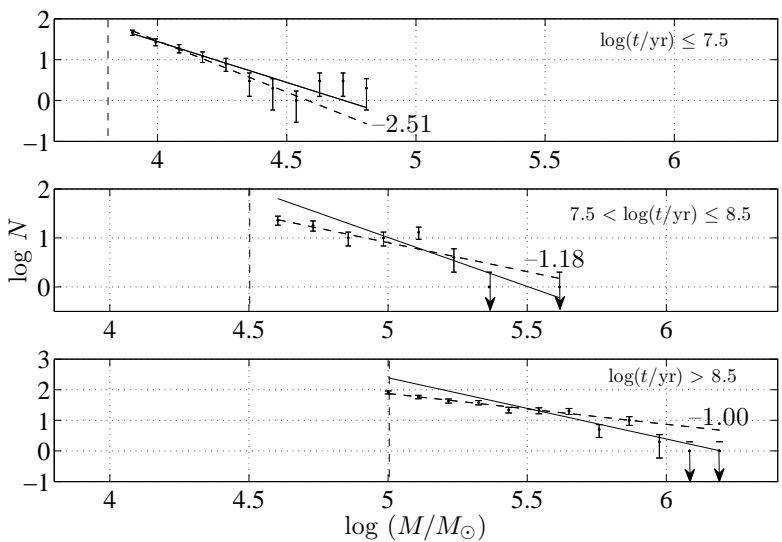

FIG. 4.- CMFs for the three different age ranges adopted in this study. The dashed lines show the best-fitting power-law CMFs to the full mass ranges available. The solid lines indicate the theoretically predicted power-law CMF. The vertical dashed lines reflect the samples' $50 \%$ completeness limits.

Our results in Fig. 4 display gradually flatter CMFs (pertaining to the full, observed mass ranges) with in- 
creasing cluster age. To distinguish between the "lowmass" and "high-mass" ranges, we divide all three samples at the mass where their CMFs exhibit a clear break in their slopes, which occurs at $\log \left(M_{\mathrm{cl}} / M_{\odot}\right) \sim$ $(4.6 \pm 0.1),(5.2 \pm 0.2)$, and $(5.7 \pm 0.2)$ for the young, intermediate-age, and old samples, respectively. We will now first discuss the intermediate-age- $\log \left(t \mathrm{yr}^{-1}\right) \in$ $[7.5,8.5]$ - and old samples, $\log \left(t \mathrm{yr}^{-1}\right) \geq 8.5$. If we only consider the less massive clusters-i.e., for the intermediate-age sample, we select the four lowest-mass bins, $\log \left(M_{\mathrm{cl}} / M_{\odot}\right) \leq 5$, and for the old sample we select the first seven bins, $\log \left(M_{\mathrm{cl}} / M_{\odot}\right) \leq 5.61$ - their distributions are (perhaps surprisingly given the expected effects of stochasticity) relatively similar, with slopes of $\alpha=(-1.18 \pm 0.43)$ and $\alpha=(-1.00 \pm$ 0.18 ) for the intermediate-age and old samples, respectively. These slopes are significantly shallower than the equivalent slope pertaining to the young sample$\log \left(t \mathrm{yr}^{-1}\right) \leq 7.5$ of $\alpha=-2.51 \pm 0.41$ for clusters with $\log \left(M_{\mathrm{cl}} / M_{\odot}\right) \leq 4.54$.

Despite their different mass ranges, the intermediateage and old samples exhibit a high similarity, i.e., relatively shallow CMFs over a small, low-mass range (presumably caused by the effects of cluster disruption; see below), combined with a steeper slope pertaining to the least-evolved, most massive clusters. For the intermediate-age sample, the fit to the CMF using the four highest-mass bins, where the effects of stochastic sampling are expected to be negligible (cf. Anders et al. $2013)-\log \left(M_{\mathrm{cl}} / M_{\odot}\right) \geq 5.0$ - yields a relatively poorly constrained slope of $\alpha \simeq-2.17$. Meanwhile, the fit to the old sample's CMF defined by the five highestmass bins- $\log \left(M_{\mathrm{cl}} / M_{\odot}\right) \geq 5.6$-indicates a slope of $\alpha=-2.21 \pm 0.42$. However, if we consider the young sample and only select its three highest-mass bins$\log \left(M_{\mathrm{cl}} / M_{\odot}\right) \geq 4.5$ - the resulting $\mathrm{CMF}$ is quite shallow, $\alpha \sim-1.0$. We note that this is likely the result of smallnumber statistics, given that the young sample contains only a handful of clusters with masses $\log \left(M_{\mathrm{cl}} / M_{\odot}\right) \geq$ 4.5. Nevertheless, the masses of the most massive clusters in the young sample define an upper envelope in Fig. 3 that extends from the youngest to the oldest ages, following a trend that is usually attributed to size-of-sample effects (e.g., Hunter et al. 2003). The lower-mass range of the young clusters' CMF exhibits a much steeper CMF that is roughly consistent with the theoretically predicted initial, unevolved CMF, characterized by $\alpha=-2$. In this context, both the intermediate-age and old samples display shallow-to-steep CMF slope changes when going from low- to high-mass clusters, which is most likely owing to the preferential disruption of the lower-mass clusters (see below).

This distinct difference with respect to the less massive clusters' CMF in the young cluster sample may indicate a mass-specific disruption law: clusters that are less massive than a critical mass are preferentially disrupted after a typical timescale on which dynamical disruption sets in. Since the cluster-formation history across the galaxy has been bursty, we cannot simply apply "standard" cluster disruption analysis (e.g., Boutloukos \& Lamers 2003; de Grijs et al. 2003c) to derive this timescale.

\subsection{Tidal-disruption processes}

While the young cluster sample exhibits an excess of relatively massive clusters (which may be owing to smallnumber statistics) - and hence displays a flatter distribution for the highest-mass range, i.e., for $\log \left(M_{\mathrm{cl}} / M_{\odot}\right) \gtrsim$ 4.5 - both the intermediate-age and old samples exhibit apparently steeper slopes consistent with $\alpha=-2$ at the high-mass end, i.e., for $\log \left(M_{\mathrm{cl}} / M_{\odot}\right) \gtrsim 5.1$ and $\log \left(M_{\mathrm{cl}} / M_{\odot}\right) \gtrsim 5.7$, respectively, than those defined by the lower-mass clusters in the respective samples. This implies that the massive star clusters in these subsamples are less significantly affected by disruption processes than their lower-mass counterparts, as expected from the mass-dependent cluster disruption formalism originally developed by Boutloukos \& Lamers (2003) and expanded upon since by a number of authors (e.g., de Grijs et al. 2003a,c; Lamers et al. 2005a,b; Gieles et al. 2005, 2007; Parmentier \& de Grijs 2008).

We first investigate if the spatial distribution of the most massive clusters in the young sample is different from those of the intermediate-age and old samples. In Fig. [5] (top) we show the spatial distribution of all young star clusters (white points), as well as that of the young star clusters with $\log \left(M_{\mathrm{cl}} / M_{\odot}\right) \geq 4.5$ (red triangles). In order to compare these to the total sample (including all detected sources), we have also included the number densities, indicated by the background colors. We find that compared with the total young cluster sample, the most massive young clusters are all located within a very compact region. In the middle and bottom panels, we display the same spatial distributions for the intermediate-age and old samples, respectively. Quantitatively, the most massive young clusters are found in a region spanning $8.3^{\prime \prime} \times 28^{\prime \prime}$ in right ascension and declination, while their intermediate-age counterparts cover an equivalent area of approximately $460^{\prime \prime} \times 83^{\prime \prime}$. The distribution of the most massive clusters - $\log \left(M_{\mathrm{cl}} / M_{\odot}\right) \geq 4.99$, red triangles - in the intermediate-age sample compared with that of their counterparts in the old sample, is significantly different; the former are dispersed around the whole galaxy. The 30 most massive clusters in the old sample are more centrally concentrated, covering a range of approximately $105^{\prime \prime} \times 20^{\prime \prime}$ (right ascension $\times$ declination); the remaining eight clusters are found throughout the galaxy's disk.

The old sample represents the potential field of the oldest galactic components. This sample contributes most clusters among all detected sources $(55 \% ; 378$ of the total of 691 clusters above the prevailing completeness threshold adopted), and hence their distribution is strongly associated with the high background number-density region. We propose the following evolutionary scenario. The oldest clusters likely formed at the time when the galaxy itself was in the process of formation, so the gravitational background experienced by this first cluster population at the time of their formation must have been very different from that in which subsequent cluster populations formed. When these latter populations formed, they found themselves inside a well-developed, centrally peaked gravitational potential, which would have strongly affected the evolution of these subsequently formed cluster populations. In the number-density color figure, the highest-density region was likely the preferential nursery of the descendants of both the intermediateage and old clusters (i.e., a nursery of both the young and intermediate-age star clusters, respectively), but in the 


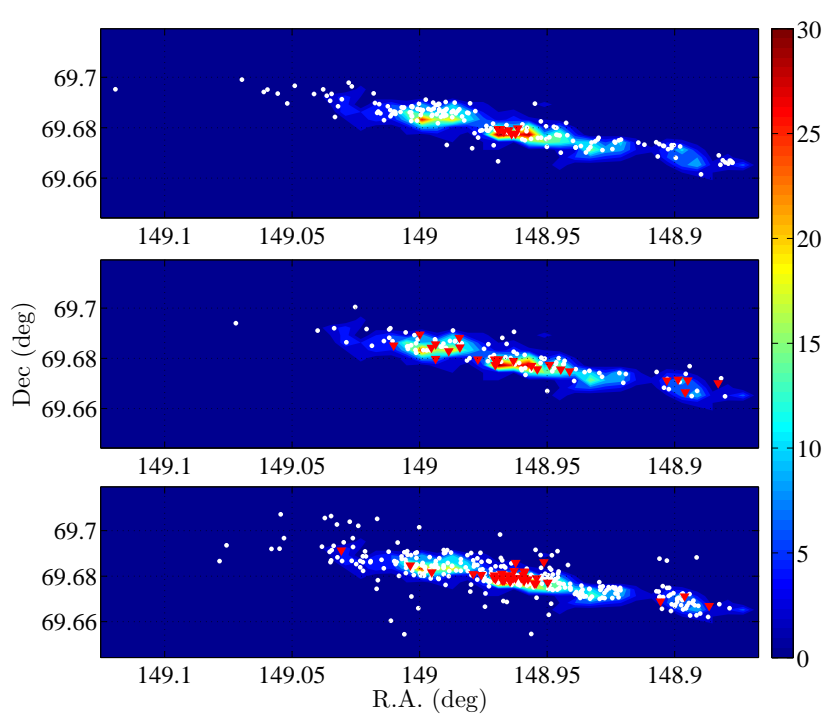

FiG. 5.- (top) Spatial distribution of the young cluster sample (white points). The most massive young clusters- $\log \left(M_{\mathrm{cl}} / M_{\odot}\right) \geq$ 4.54 - are indicated by red triangles; the background colors represent the number density distribution of all detected sources. (middle and bottom) Same as the top panel, but for the intermediateage and old samples, respectively.

mean time, the strong tidal field in the highest-density region will also have tidally truncated and stripped off the outer layers of the largest clusters, thus only leaving the more compact clusters as survivors.

In order to test if this proposed scenario is viable, we explored the size distribution of the surviving, most massive young clusters. If those young massive star clusters really have been affected by tidal disruption, they should be significantly smaller than their counterparts that are located far beyond the immediate influence of the central tidal field. We used the ISHAPE software package (Larsen 1999) to determine the cluster sizes. This package is used to estimate the intrinsic shape parameters of extended objects given the observational point-spread function. It attempts to fit the radial profiles of extended sources with simple analytical functions. Here, we are mainly concerned with the observed clusters' empirical sizes. The derived effective radii are not very sensitive to the choice of model (Larsen 1999). A related result was obtained by Kundu \& Whitmore (1998), who found that effective radii derived from fits to a King (1962) model were also quite insensitive to the adopted concentration parameter $c \equiv \log \left(r_{\mathrm{t}} / r_{0}\right)$, where $r_{\mathrm{t}}$ is the tidal radius and $r_{0}$ the King radius. We adopted a King model with $c=5$ to fit the clusters' radial profiles. Finally, we determined the clusters' half-light radii $\left(R_{\mathrm{hl}}\right)$ as an appropriate representation of their sizes, in units of pixels 7

Figure 6] (top) displays the $\log \left(M_{\mathrm{cl}} / M_{\odot}\right)$ versus $R_{\mathrm{hl}}$ diagram for all young star clusters. The dashed line indicates the critical mass we adopted for the most massive

\footnotetext{
7 Size comparisons for clusters of different ages may introduce a bias because of mass segregation (de Grijs et al. 2002a,b,c), however, which will cause $R_{\mathrm{hl}}$ to decrease as a star cluster evolves. At the same time, their half-mass radii will increase, so that for relatively old sample clusters, we may overestimate their average densities.
}

clusters in the young sample. An apparent mean smaller size for these most massive clusters, $\left\langle R_{\mathrm{hl}}\right\rangle \simeq 2.6 \mathrm{pc}$, is found, as well as a smaller size spread: the less massive clusters have a mean half-light radius of $\left\langle R_{\mathrm{hl}}\right\rangle \simeq 4.3$ pc. We also calculated the clusters' average density, $\rho=M_{\mathrm{cl}} /\left(2 R_{\mathrm{hl}}^{3}\right)$ (see the background colors), where we simply assumed that the clusters' half-light radii are roughly equal to their half-mass radii $\left(R_{\mathrm{hm}}\right)$. It thus appears that the most massive young clusters are also the highest-density, most compact objects. Note that despite the relatively small sample size of eight objects above the critical mass limit, these eight sources are the brightest clusters in the young subsample. In Fig. 3. they are found well above our galaxy-wide selection limit. If the young sample had included additional, more extended objects of similar masses, our procedures would, without a doubt, have detected such objects. This is supported by the fact that the young sample does include more extended objects below the critical mass limit, which are less luminous (by definition, given their very similar ages) than the higher-mass objects we would have missed. As such, we conclude that there is indeed a tantalizing hint that the most massive young clusters are systematically smaller than their lower-mass counterparts. (Of course, this argument hinges on the basic underlying assumption that the clusters' radial profiles do not differ significantly among the sample objects.)

In Fig. 6 (middle and bottom), we show the same distributions as in Fig. 6] (top), but for the intermediateage and old samples. This time, however, we do not observe any clearly smaller mean size for the most massive clusters: for the intermediate-age clusters, the mean half-light radii for the low- and high-mass subsamples are $\left\langle R_{\mathrm{hl}, \text { low }}\right\rangle \simeq 4.3 \mathrm{pc}$ and $\left\langle R_{\mathrm{hl}, \text { high }}\right\rangle \simeq 4.2 \mathrm{pc}$, respectively. The equivalent sizes for the the old sample are $4.1 \mathrm{pc}$ and $4.3 \mathrm{pc}$, respectively. This may indicate that the remaining massive star clusters in the intermediate-age and old samples are not as strongly affected by the prevailing tidal field, in part because of their more extended distributions, i.e., away from the peak of the tidal field's distribution. This is a reasonable conclusion, since the first generation of star clusters (the old sample) likely formed when the galaxy itself was still forming, so that (i) a strong tidal field may not yet have been established, and (ii) the remaining old clusters have proved robust with respect to the effects of the prevailing tidal field. We are thus likely observing an old cluster population that has survived the potentially devastating effects of tidal stripping. Note, however, that although the intermediateage, most massive clusters are more dispersed than their counterparts in the young sample, which should not yet have been affected strongly by the tidal field, some may already have been disrupted.

Alternatively, the reason why the massive old clusters may still be confined to a relatively small volume in and near the galactic center region might be found in the complex structure of the M82 disk. It is well-known that the inner $\sim 1 \mathrm{kpc}$ of M82 is dominated by a stellar bar (e.g., Wills et al. 2000) in solid-body rotation. The phase mixing might be slow enough for the bar to retain its identity over a sufficient time so as to remain self-constrained (J. S. Gallagher, private communication). In addition, since the density in the region is high, simple calculations imply that the area's self-gravity is non-negligible compared 
with the rotational shear, therefore also prohibiting a rapid dispersion of the central starburst region.

Finally, we note that the old clusters survived the recent tidal encounter with M81, which may have occurred between approximately 150 Myr and 1 Gyr ago (e.g., Brouillet et al. 1991; Yun et al. 1994; de Grijs et al. 2001, 2003c; Smith et al. 2007). Although this might appear curious at first sight, we point out that our old cluster sample only includes objects that are more massive than a few $\times 10^{4} M_{\odot}$, while disruption - both owing to internal relaxation and caused by external perturbations - predominantly affects the lower-mass clusters in a given sample. However, because of the age-dependent selection limit, we cannot make any definite statements about whether or not the last tidal encounter with M82 may have led to significantly enhanced cluster disruption among the lower-mass clusters in the oldest subsample (but, for a similar scenario in the context of the interacting galaxy M51, see Lamers et al. 2005a).

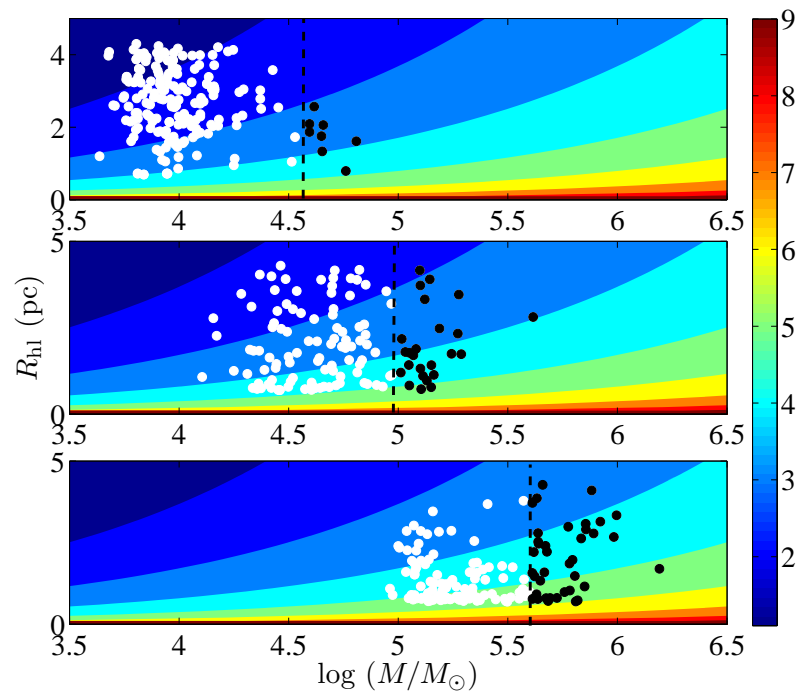

Fig. 6.- (top) $\log \left(M_{\mathrm{cl}} / M_{\odot}\right)$ versus $R_{\mathrm{hl}}$ diagram for the young star clusters. The vertical dashed line indicates the critical mass adopted for the most massive clusters, i.e., $\log \left(M_{\mathrm{cl}} / M_{\odot}\right) \geq 4.54$, while the background colors and contours indicate the cluster population's density distribution. (middle and bottom) Same as the top panel, but for our intermediate-age and oldest samples, respectively.

\section{CONCLUSION}

Using high-resolution HST images based on multiplepassband observations, we selected a complete sample of
846 star clusters in the disk of M82, for which we determined the respective magnitudes, as well as their age, mass, and size information. We aimed at characterizing the effects of cluster disruption based on a detailed analysis of the clusters' age and mass distributions. Our primary results can be summarized as follows.

1. The young sample- $\log \left(t \mathrm{yr}^{-1}\right) \leq 7.5$ - displays a steep-to-shallow CMF slope change when going from low to high cluster masses, with a characteristic break near $\log \left(M_{\mathrm{cl}} / M_{\odot}\right)=4.6$, while both the intermediate-age- $\log \left(t \mathrm{yr}^{-1}\right) \in[7.5,8.5]$ - and old samples- $\log \left(t \mathrm{yr}^{-1}\right) \geq 8.5$ - display a shallowto-steep slope change in the same direction. This distinct difference with respect to the less massive clusters' CMF in the young cluster sample may indicate a mass-specific disruption law: clusters that are less massive than a critical mass are preferentially disrupted on a typical, mass-dependent timescale $\left(t_{\text {dis }} \gg 30 \mathrm{Myr}\right)$.

2. Compared with the total young cluster sample, the most massive young clusters are all located within a very compact spatial region. The distribution of the most massive clusters in the intermediateage sample $-\log \left(M_{\mathrm{cl}} / M_{\odot}\right) \geq 4.99$ - compared with that of their counterparts in the young sample, is markedly different; the latter are dispersed around the whole galaxy. The 30 most massive clusters in the old sample are significantly more centrally concentrated.

3. It is clear that the most massive young clusters are also the highest-density, most compact objects. This result hence strongly suggests that the properties of the most massive young clusters are affected by the galaxy's tidal field. For the intermediateage and old samples, we do not observe any clearly smaller size for the most massive clusters. This may indicate that we are observing a different subset of the initial cluster population for each of the age ranges (i.e., differently affected by tidal effects and evolution), and that the remaining, surviving massive star clusters in the intermediate-age and old samples are - on the whole - more robust with respect to changes due to the prevailing tidal field.

\section{ACKNOWLEDGMENTS}

This article forms part of the first author's requirements to obtain an M.Sc. degree at Peking University. We thank Chaojian Wu for technical assistance with programming issues. We acknowledge research support from the National Natural Science Foundation of China (grants 11073001 and 11373010).

\section{REFERENCES}

Anders, P., Bissantz, N., Fritze-v. Alvensleben, U., \& de Grijs, R. 2004, MNRAS, 347, 196

Anders, P., Kotulla, R., de Grijs, R., \& Wicker, J. 2013, ApJ, 778,138

Barker, S., de Grijs, R., \& Cerviño, M. 2008, A\&A, 487, 711

Bastian, N., Gieles, M., Ercolano, B., \& Gutermuth, R. 2009, MNRAS, 392, 868

Bastian, N., Weisz, D. R., Skillman, E. D., et al. 2011, MNRAS, 412,1539
Boutloukos, S. G., \& Lamers, H. J. G. L. M. 2003, MNRAS, 338, 717

Bressert, E., Bastian, N., Gutermuth, R., et al. 2010, MNRAS, 409, L54

Brouillet, N., Baudry, A., Combes, F., Kaufman, M., \& Bash, F. 1991, A\&A, 242, 35

Cantiello, M., Brocato, E., \& Blakeslee, J. P. 2009, A\&A, 503, 87

Chandar, R., Fall, S. M., \& Whitmore, B. C. 2010, ApJ, 711, 1263

Chernoff, D. F., \& Shapiro, S. L. 1987, ApJ, 322, 113 
Chernoff, D. F., \& Weinberg, M. D. 1990, ApJ, 351, 121

de Grijs, R., O'Connell, R. W., \& Gallagher, J. S. 2001, AJ, 121, 768

de Grijs, R., Johnson, R. A., Gilmore, G. F., \& Frayn, C. M 2002a, MNRAS, 331, 228

de Grijs, R., Gilmore, G. F., Johnson, R. A., \& Mackey, A. D. 2002b, MNRAS, 337, 597

de Grijs, R., Gilmore, G. F., Mackey, A. D., et al. 2002, MNRAS, 337,597

de Grijs, R., Anders, P., Bastian, N., et al. 2003a, MNRAS, 343 1285

de Grijs, R., Fritze-v. Alvensleben, U., Anders, P., et al. 2003b, MNRAS, 342, 259

de Grijs, R., Bastian, N., \& Lamers, H. J. G. L. M. 2003c, MNRAS, 340, 197

de Grijs, R., Parmentier, G., \& Lamers, H. J. G. L. M. 2005 MNRAS, 364, 1054

de Grijs, R., \& Parmentier, G. 2007, ChJAA, 7, 155

de Grijs, R., \& Anders, P. 2012, ApJL, 758, L22

de Grijs, R., Anders, P., Zackrisson, E., \& Östlin, G. 2013, MNRAS, 431, 2917

de Wit, W. J., Testi, L., Palla, F., \& Zinnecker, H. 2005, A\&A, 437,247

Gieles, M., Bastian, N., Lamers, H. J. G. L. M., \& Mout, J. N. 2005, A\&A, 441, 949

Gieles, M., Lamers, H. J. G. L. M., \& Portegies Zwart, S. F. 2007 ApJ, 668, 268

Gieles, M., Bastian, N., \& Ercolano, B. 2008, MNRAS, 391, L93

Gies, D. R. 1987, ApJS, 64, 545

Goodwin, S. P. 1997, MNRAS, 286, 669

Gvaramadze, V. V., \& Bomans, D. J. 2008, A\&A, 490, 1071

Hunter, D. A., Elmegreen, B. G., Dupuy, T. J., \& Mortonson, M. 2003, AJ, 126, 1836

Hwang, N., \& Lee, M. G. 2008, AJ, 135, 1567

Hwang, N., \& Lee, M. G. 2010, IAU Symp., 266, 423

King, I. 1962, AJ, 67, 471

Konstantopoulos, I. S., Bastian, N., Smith, L. J., et al. 2008, ApJ, 674, 846

Kotulla, R., Fritze, U., Weilbacher, P., \& Anders, P. 2009, MNRAS, 396, 462

Kouwenhoven, M. B. N., Goodwin, S. P., de Grijs, R., Rose, M., \& Kim, S. S. 2014, MNRAS, 445, 2256

Krist, J. E., \& Hook, R. N. 1997, HST Calibration Workshop with a New Generation of Instruments, Casertano, S., Jedrzejewski, R., Keyes, T., \& Stevens, M., eds., (Baltimore: STScI), p. 192

Kruijssen J. M. D. 2012, MNRAS, 426, 3008

Kundu, A., \& Whitmore, B. C. 1998, AJ, 116, 2841

Lada, C. J., \& Lada, E. A. 2003, ARA\&A, 41, 57

Lamers, H. J. G. L. M., Gieles, M., \& Portegies Zwart, S. F. 2005, A\&A, 429, 173

Lamers, H. J. G. L. M., Gieles, M., Bastian, N., et al. 2005, A\&A 441, 117

Lançon A., Gallagher J.S., III, Mouhcine M., et al. 2008, A\&A, 486,165

Larsen, S. S. 1999, A\&AS, 139, 393
Larsen, S. S. 2002, AJ, 124, 1393

Larsen, S. S., de Mink, S. E., Eldridge, J. J., et al. 2011, A\&A 532,147

Lim, S., Hwang, N., \& Lee, M. G. 2013, ApJ, 766, 20

Longmore, S. N., Kruijssen, J. M. D., Bastian, N., et al. 2014, Protostars and Planets, VI, in press (arXiv:1401.4175)

Mayya, Y. D., Romano, R., Rodríguez-Merino, L. H., et al. 2008, ApJ, 679, 404

Melo, V. P., Muñoz-Tuñón, C., Maíz-Apellániz, J., et al. 2005, ApJ, 619, 270

Mengel, S., Lehnert, M. D., Thatte, N., \& Genzel, R. 2002, A\&A, 383,137

Meurer, G. R., Heckman, T. M., Leitherer, C., et al. 1995, ApJ, 110,2665

Miller, G. E., \& Scalo, J. M. 1979, ApJS, 41, 513

Mutchler, M., Bond, H. E., Christian, C. A., et al. 2007, PASP, 119,1

O'Connell, R. W., \& Mangano, J. J. 1978, ApJ, 221, 62

O'Connell, R. W., Gallagher, J. S., Hunter, D. A., \& Colley, W. N. 1995, ApJ, 446, 1

Ohyama, Y., Hamana, T., Kashikawa, N., et al. 2002, AJ, 123, 2903

Parker, R. J., \& Goodwin, S. P. 2007, MNRAS, 380, 1271

Parmentier, G., \& de Grijs, R. 2008, MNRAS, 383, 1103

Pellerin, A., Meurer, G. R., Bekki, K., et al. 2010, AJ, 139, 1369

Pflamm-Altenburg, J., \& Kroupa, P. 2010, MNRAS, 404, 1454

Portegies Zwart, S. F., McMillan, S. L. W., \& Gieles, M. 2010, ARA\&A, 48, 431

Scheepmaker, R. A., Haas, M. R., Gieles, M., et al. 2007, A\&A, 469,925

Schilbach, E., \& Röser, S. 2008, A\&A, 489, 105

Shopbell, P. L., \& Bland-Hawthorn, J. 1998, ApJ, 493, 129

Silva-Villa, E., \& Larsen, S. S. 2011, A\&A, 529, A25

Smith, L. J., \& Gallagher, J. S. 2001, MNRAS, 326, 1027

Smith, L. J., Westmoquette, M. S., Gallagher, J. S., O'Connell,

R. W., Rosario, D. J., \& de Grijs, R. 2006, MNRAS, 370, 513

Smith, L. J., Bastian, N., Konstantopoulos, I. S., et al. 2007, ApJL, 667, L145

Stevens, I. R., Read, A. M., \& Bravo-Guerrero, J., et al. 2003, MNRAS, 343, 47

Strickland, D. K., \& Heckman, T. M. 2007, ApJ, 658, 258

Takahashi, K., \& Portegies Zwart, S. F. 2000, ApJ, 535, 759

Westmoquette, M. S., Smith, L. J., Gallagher, J. S., III, et al. 2007, ApJ, 671, 358

Whitmore, B. C., Zhang, Q., Leitherer, C., Fall, M., Schweizer, F., \& Miller, B. W. 1999, AJ, 118, 1551

Whitmore, B. C., Chandar, R., Schweizer, F., et al. 2010, AJ, 140,75

Wills, K. A., Das, M., Pedlar, A., Muxlow, T. W. B., \& Robinson, T. G. 2000, MNRAS, 316, 33

Yun, M. S., Ho P. T. P., \& Lo K. Y. 1994, Natur., 372, 530

Zhang, Q., \& Fall, M. 1999, ApJ, 527, 81 